\title{
OPEN Noble metal alloy thin films by atomic layer deposition and rapid Joule heating
}

\author{
Yuanyuan Guo ${ }^{1}$, Yiming Zou ${ }^{1}$, Chunyu Cheng ${ }^{1}$, Leyan Wang ${ }^{1}$, Riko I Made ${ }^{2}$, Ronn Goei ${ }^{1}$, \\ Kwan Wee $\mathrm{Tan}^{1}{ }^{1}$, Shuzhou $\mathrm{Li}^{1 \otimes}$ \& Alfred ling Yoong Tok ${ }^{1 凶}$
}

Metal alloys are usually fabricated by melting constituent metals together or sintering metal alloy particles made by high energy ball milling (mechanical alloying). All these methods only allow for bulk alloys to be formed. This manuscript details a new method of fabricating Rhodium-Iridium (Rh-Ir) metal alloy films using atomic layer deposition (ALD) and rapid Joule heating induced alloying that gives functional thin film alloys, enabling conformal thin films with high aspect ratios on 3D nanostructured substrate. In this work, ALD was used to deposit Rh thin film on an $\mathrm{Al}_{2} \mathrm{O}_{3}$ substrate, followed by an Ir overlayer on top of the Rh film. The multilayered structure was then alloyed/ sintered using rapid Joule heating. We can precisely control the thickness of the resultant alloy films down to the atomic scale. The Rh-Ir alloy thin films were characterized using scanning and transmission electron microscopy (SEM/TEM) and energy dispersive spectroscopy (EDS) to study their microstructural characteristics which showed the morphology difference before and after rapid Joule heating and confirmed the interdiffusion between $\mathrm{Rh}$ and Ir during rapid Joule heating. The diffraction peak shift was observed by Grazing-incidence X-ray diffraction (GIXRD) indicating the formation of RhIr thin film alloys after rapid Joule heating. X-ray photoelectron spectroscopy (XPS) was also carried out and implied the formation of Rh-Ir alloy. Molecular dynamics simulation experiments of Rh-Ir alloys using Large-Scale Atomic/Molecular Massively Parallel Simulator (LAMMPS) were performed to elucidate the alloying mechanism during the rapid heating process, corroborating the experimental results.

Alloys such as steels and bronze have traditionally been applied in engineering applications due to their significantly improved physical and chemical properties as compared to their monometallic counterparts. Many of these applications focus on properties such as high oxidation and corrosion resistance ${ }^{1,2}$, superior wear and fatigue resistance ${ }^{3}$, high thermal stability ${ }^{1,4}$ and excellent electrocatalytic ability ${ }^{5,6}$. Recently, modern alloys have found new applications in industries like biomedical, aerospace industries and energy conversion and storage applications ${ }^{7,8}$. Conventional methods used to fabricate metal alloys typically result in bulk alloys; even with methods like mechanical alloying ${ }^{9,10}$ which give nanostructured bulk alloys that have drawbacks like precisely control of chemical composition, thickness and microstructure.

Thin film deposition techniques like physical vapor deposition (PVD) $)^{11,12}$, chemical vapor deposition (CVD) and Atomic Layer Deposition (ALD) ${ }^{13-16}$ have been used to prepare alloy thin films. PVD is a deposition process using an alloy source, but it is limited by directionality and conformality constraints. CVD is challenging for high purity, ultra-thin high aspect ratio films, because the process is not self-limiting, nor can it fabricate films on high aspect ratio substrates. ALD allows high purity, highly conformal on high aspect ratio, 3D patterned substrates at low temperature deposition controlled at the atomic scale. These techniques have been widely used in areas like semiconductor manufacturing, fuel cells, solar cells and batteries due to the high surface area, rich reaction sites of the thin film materials compared to bulk materials. As the requirements for these advanced devices become more stringent, the fabrication methods will be required to deposit conformal and uniform thin films with precisely controlled chemical composition, thickness and surface roughness, on substrates with 3D high aspect ratio surface feature ${ }^{12,17,18}$ all the known techniques today are unable to fabricate such thin film alloys.

Typically, metal alloys are post-processed at high temperatures to ensure uniform atomic mixing and formation of functional crystalline phases. Compared to other heating methods for alloy preparation (spark plasma

${ }^{1}$ School of Materials Science and Engineering, Nanyang Technological University, Singapore 639798, Singapore. ${ }^{2}$ Institute of Materials Research and Engineering, A*STAR (Agency for Science Technology and Research), Singapore 138634, Singapore. ${ }^{\square}$ email: lisz@ntu.edu.sg; MIYTok@ntu.edu.sg 


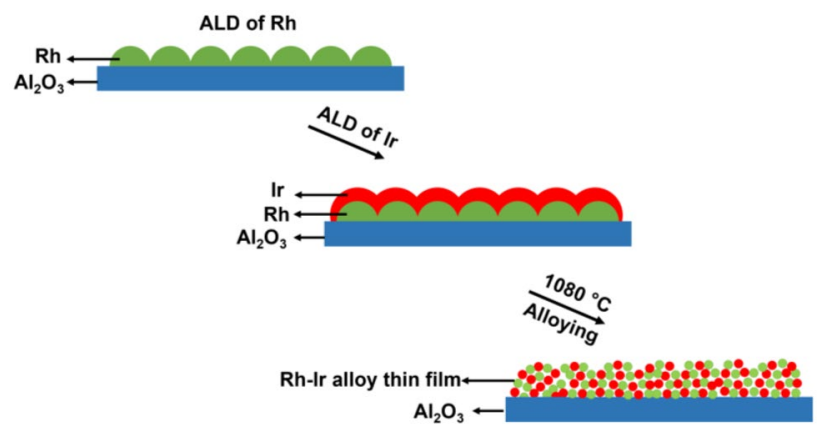

Figure 1. Schematic illustration of the fabrication process of Rh-Ir alloy thin film.

sintering, pressure less sintering, hot press sintering, microwave sintering), electric Joule heating (EJH) has the advantages of ultrafast heating and rapid cooling (on the orders of $10^{3}-10^{5} \mathrm{~K} / \mathrm{s}$ ), allowing precise control of homogeneous elemental composition and distribution as well as nonequilibrium transformation of single-phase alloys by inhibiting phase separation ${ }^{19,20}$. allowing precise control of homogeneous elemental composition and distribution as well as nonequilibrium transformation of single-phase alloys by inhibiting phase separation. It has also been reported that atomic interdiffusion distance is shorter in smaller particle sized metals as compared to the bulk at the same temperature ${ }^{21}$. Similarly, metal thin films with nanometer thickness could also exhibit this same behaviour, where the metal atoms would diffuse fast between the constituent layers ${ }^{21}$. In addition, their melting point would be much lower than that of the corresponding bulk metals due to their nanometer thickness ${ }^{22}$. As such, metallic alloys could be fabricated using alternating layers of thin film constituent metals.

In this work, we demonstrate for the first time a novel strategy consisting of ALD to fabricate alternating layers of nanometer-thin constituent Ir and Rh films on the basis of crystallographic similarity, and subsequent rapid EJH treatment to alloy \& sinter these $\mathrm{Rh}$-Ir films into an alloy. After ALD, the multilayered structure was alloyed by EJH at $1080^{\circ} \mathrm{C}$ for $5 \mathrm{~s}$ to obtain Rh-Ir alloy thin film (Fig. 1). Characterization of the alloy thin film was performed using GIXRD, XPS, TEM and SEM. Molecular Dynamics simulation experiments were employed to elucidate the melting and alloying mechanisms of Rh-Ir alloy thin film.

\section{Experimental section}

ALD of Rh on sapphire. A custom-built ALD reactor was used to fabricate the thin films on a $1 \times 1 \mathrm{~cm}^{2}$ sapphire $\left(\alpha-\mathrm{Al}_{2} \mathrm{O}_{3}(0001)\right)$ substrate. ALD precursors used for Rh were Rhodium (III) acetylacetonate and ozone as a co-reactant generated in an ozone generator (Nanofrontier, XLK-G20) from high purity oxygen (99.999\%). A base pressure was kept at $1.0 \mathrm{mbar}$ by applying $150 \mathrm{sccm}$ nitrogen gas passing through the main chamber. The substrate temperature was kept at $210^{\circ} \mathrm{C}$ and the sublimation temperature of Rh precursor was $150{ }^{\circ} \mathrm{C} .500$ cycles were carried out for Rh ALD. Each cycle was expressed as $\mathrm{t} 1-\mathrm{t} 2-\mathrm{t} 4-\mathrm{t} 1-\mathrm{t} 2-\mathrm{t} 4-\mathrm{t} 3-\mathrm{t} 4$ where $\mathrm{t} 1$ is the pulse of carrier gas into the precursor holder $(5000 \mathrm{~ms}), \mathrm{t} 2$ is the pulse of precursor $(2000 \mathrm{~ms})$, $\mathrm{t} 3$ is the pulse of ozone $(300 \mathrm{~ms})$ and $t 4$ is the purging time $(2000 \mathrm{~ms})$. A more detailed description of this ALD process conditions and parameters can be found in our previous paper $^{23}$.

ALD of Ir on Rh. The as prepared Rh on sapphire was loaded in the reaction chamber as substrate and the temperature was kept at $180^{\circ} \mathrm{C}$. ALD precursor used for Ir was Iridium (III) acetylacetonate, and ozone as a coreactant $t^{24}$. The sublimation temperature of Ir precursor was $180^{\circ} \mathrm{C}$. The other operation condition and deposition parameters including cycle number, deposition sequence and pulse/purge times of each reactant were same as used for ALD of $\mathrm{Rh}^{23}$.

Rapid Joule heating of Rh-Ir thin film. The EJH experiments were using a custom-built setup as reported in literature ${ }^{19-23,25}$ under low vacuum conditions ( $<1$ Torr). The ALD Rh-Ir thin films on sapphire substrates were placed on top of a carbon paper support (Fuel Cell Earth). A tuneable source meter was used to supply potential and current values up to $30 \mathrm{~V}$ and $20 \mathrm{~A}$, respectively, to heat the carbon paper. The thin film samples were Joule-heated with a ramp dwell of $3 \mathrm{~s}$ and then held at the respective temperatures for $5 \mathrm{~s}$. Finally, the samples were cooled naturally to ambient temperature over $5 \mathrm{~min}$. The peak temperatures of the carbon paper were obtained using a Raytek Raynger thermal infrared sensor.

Characterization. SEM images were obtained with a field emission gun source (JEOL, 7600F, FESEM) and (ThermoFisher Quatro FE-ESEM). SEM EDX Maps were collected with $20 \mathrm{keV}$ electron energy, to avoid sample damage and carbon deposition, signal collected with short dwell time and multiple frames, i.e. $-10 \mu$ s and 1000 frames. TEM imaging and EDX mapping were carried out using JEM-2100F (JEOL, Japan), operated at $200 \mathrm{kV}$, equipped with Oxford EDS Detector. TEM sample were prepared by focused ion beam (FIB) technique, using FEI Nova 600 Nanolabs Dual Beam. The chemical composition and binding information analysis were performed by X-ray photoelectron spectroscopy (Shimadzu Kratos Axis Supra, XPS with $15 \mathrm{kV}$ and $15 \mathrm{~mA} \mathrm{Al} \mathrm{Ka}$ source). The binding energy scale was calibrated automatically with standard samples (silver and gold). The raw 
XPS data analysis was finished by ESCApe software and the binding energy peak positions were calibrated with $\mathrm{C} 1 \mathrm{~s}$ peak at $284.8 \mathrm{eV}$. Shirley background and Gaussian-Lorentzian function with $30 \%$ Gaussian peak profile were applied for XPS peak fitting. The crystalline structure of the films was examined by grazing incidence X-ray diffraction (Bruker D8 discover XRD System, GI-XRD) using a Cu-Ka radiation with a wavelength of $1.54 \AA$ and a fixed grazing angle $\left(1.0^{\circ}\right)$. The XRR curve was fitted by the software Leptos based on various parameters, including thickness, density, roughness and crystal structure of the thin films.

Computational details. Rh-core-Ir-shell nanoparticles (Rh-Ir NP) were constructed from a large cubic fcc single crystal to simulate the alloying process of Rh-Ir film. The total number of atoms was 8247 which corresponds to a radius of about $3.1 \mathrm{~nm}$. The $\mathrm{Rh}$ percentage is 66.4 at.\% according to the experimental results.

Molecular dynamics (MD) simulations were performed using the large-scale atomistic/molecular massively parallel simulator (LAMMPS) open source code ${ }^{26,27}$. The embedded atom method (EAM) potentials ${ }^{28}$ were adopted to depict the interatomic interactions, which describe the lattice parameter, cohesive energy, surface energy, elastic constant, and thermodynamic as well as transitional properties of metals as well as their alloys. The total potential energy of EAM potential is written ss $^{29}$ :

$$
\mathrm{E}_{i}=\mathrm{F}_{i} \sum_{i \neq j} \rho_{i j}\left(r_{i j}\right)+\sum_{i \neq j} \varphi_{i j}\left(r_{i j}\right)
$$

where $E_{i}$ and $F_{i}$ are cohesive and embedding energies of atom $i$, respectively. $\rho_{i j}\left(r_{i j}\right)$ and $\varphi_{i j}\left(r_{i j}\right)$ are the electron density and pair interaction of $j$ atoms located around the $i$ atom at distance $r_{i j}$.

Firstly, the Rh-Ir NP was first relaxed to a local minimum energy state. After full relaxation, the Rh-Ir NP was heated and constant temperature was employed to allow energy fluctuations. A Nose-Hoover thermostat ${ }^{30}$ was used to control the vibrational temperature for every step. Pressure was not controlled. The Rh-Ir NP was heated including a series of simulations over a temperature range of $27-1927^{\circ} \mathrm{C}$ in incremental of $100{ }^{\circ} \mathrm{C}$ at a heating rate of $0.5^{\circ} \mathrm{C} / \mathrm{ps}$. In addition, a smaller step of $20^{\circ} \mathrm{C}$ was employed to study the alloying behaviour more accurately around the melting point. At each temperature, the simulations were carried out for $100 \mathrm{ps.}$

To further understand the alloying mechanism during the heating process, the Lindemann index was carried out. It rises linearly with the increased temperature in the initial heating stage, and a sharp jump around the melting point is presented. For a system of $\mathrm{N}$ atoms, the Lindemann index for the ith atom is written as ${ }^{31}$ :

$$
\delta_{i}=\frac{1}{N-1} \sum_{j \neq 1} \frac{\sqrt{\left\langle R_{i j}^{2}\right\rangle-\left\langle R_{i j}\right\rangle^{2}}}{\left\langle R_{i j}\right\rangle}
$$

and the system-averaged Lindemann index is defined as:

$$
\delta=\frac{1}{N} \sum_{i} \delta_{i}
$$

where $R_{i j}$ is the distance between the ith and jth atoms. The brackets \langle\rangle is the ensemble average at the temperature.

Lindemann index (correlation of the distance between atoms) was used to identify the melting process of $\mathrm{Rh}-\mathrm{Ir}$ particle. At low temperature (below melting point), Lindemann index increases linearly and slowly with temperature. While around the melting temperature, the index is expected to jump suddenly because of the increased movement of atoms. In this work, the melting process of $\mathrm{Rh}-\mathrm{Ir}$ particle will be investigated by studying the variation of the Lindemann index with temperature.

\section{Results and discussion}

Figure 1 shows a schematic of the ALD deposition process and EJH for the Rh-Ir alloy thin film fabrication. Rh was deposited as the first layer on $\mathrm{Al}_{2} \mathrm{O}_{3}$ substrate with a thickness of $20 \mathrm{~nm}$ as measured by X-ray reflectivity (XRR), followed by the deposition of $10 \mathrm{~nm}$ thick Ir overlayer film on the Rh film surface. After that, Rh-Ir alloy thin film was obtained after a EJH process for $5 \mathrm{~s}$ at temperature around $1080^{\circ} \mathrm{C}$.

SEM was employed to understand the morphology of the as-prepared Rh-Ir thin film before and after EJH. As shown in Fig. 2, the Rh-Ir thin film has spherical morphology before and after EJH. The particle size before $\mathrm{EJH}$ is $10.0 \pm 3.3 \mathrm{~nm}$ based on measurements of at least 100 individual Rh-Ir particles by the SEM image analysis software, whereas the size after EJH induced alloying is larger $(31.9 \pm 7.7 \mathrm{~nm})$, likely due to the atomic migration, and grain growth ${ }^{32}$. Classic sintering behaviour can be observed, where particles consolidate to form bigger particles (Ostwald ripening) and some voids ${ }^{33}$.

The EDX maps in Fig. 3 show that the constituent elements of the Rh-Ir thin film are homogenously distributed before and after EJH. We did not observe Rh or Ir segregation, which might indicate the formation of an alloy (Fig. 3). On the other hand, X-ray signal from the alloyed sample is significantly higher than the pre-alloyed sample, leading to two possible scenarios. First, the film might become denser after alloying thus improving the electron beam (e-beam) and film interaction. The increase in e-beam-film interaction would then increase the X-ray yield. At the same time, a denser film would also leave localized voids (see Fig. $2 \mathrm{~b}$ ) that allow for X-rays from the substrates to escape easier, reaching the detector and ultimately recorded as brighter. Monte Carlo simulation using CASINO software ${ }^{34}$ showed that $20 \mathrm{keV}$ electron penetrates both $\mathrm{Rh}$ and Ir film easily and with minimum interaction. Most of the interaction volume happened at about $1.1 \mathrm{um}$ from the film surface, that is probing deeply into the sapphire substrates. The second scenario is Rh-Ir interdiffusion, where 

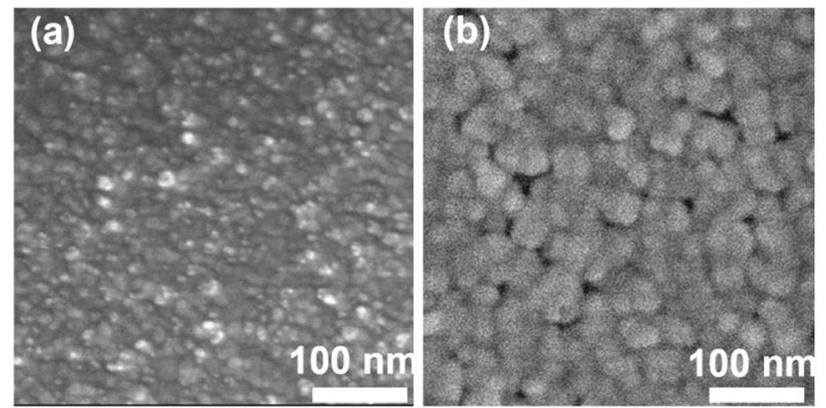

Figure 2. Top-view SEM image of Rh-Ir thin film: (a) before EJH; (b) after EJH.
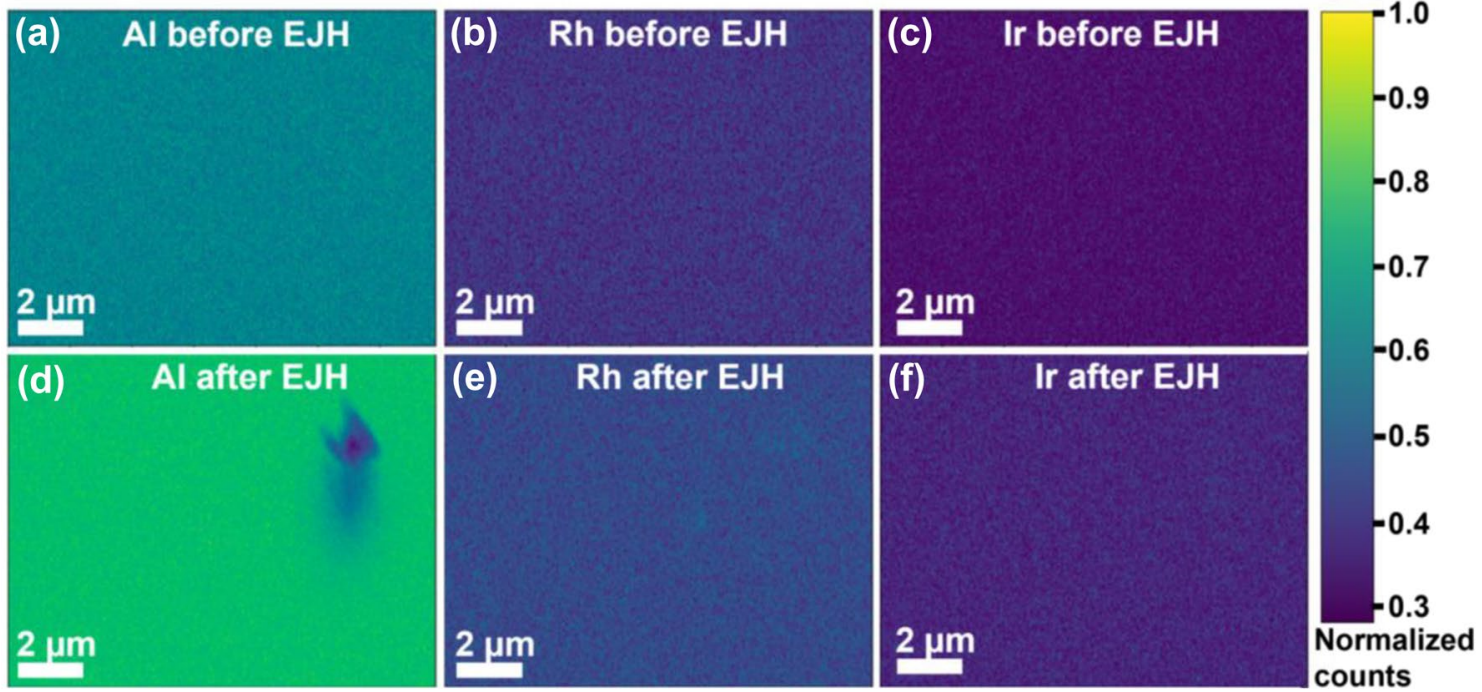

Figure 3. Low magnification EDX elemental maps of Rh-Ir thin film: (a-c) before EJH; (d-f) after EJH.
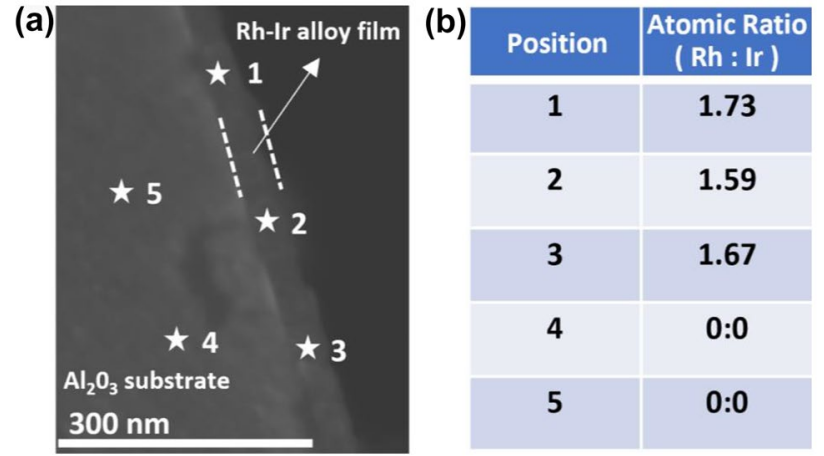

Figure 4. (a) Cross-sectional SEM image and (b) point elemental analysis of $\mathrm{Rh}$-Ir alloy thin film on $\mathrm{Al}_{2} \mathrm{O}_{3}$ substrate.

Rh diffuses up to the surface and Ir diffuses down towards the substrate. Traditionally, for bulk constituent layers, this scenario would be less likely, as it only possible for a limited range of composition (less than $40 \mathrm{wt}$.\% Rh or more than $90 \mathrm{wt} . \% \mathrm{Rh}$ ), and thermodynamically $\mathrm{Rh}-\mathrm{Ir}$ system are stable as solid solution at temperature more than $1335^{\circ} \mathrm{C}^{35}$. However, as our thin films are only $10-20 \mathrm{~nm}$ thickness, we postulate that the atomic interdiffusion distance of Ir and Rh can be similarly reduced as observed in nanoparticles ${ }^{21}$, thereby promoting $\mathrm{Rh}-\mathrm{Ir}$ interdiffusion to form a more uniform atomic composition in the alloy film and is the most likely cause of the brighter EDX signals observed.

Representative elemental compositions at random positions of the $\mathrm{Rh}$-Ir alloy thin film were investigated by point EDX analyses, respectively (Fig. 4). Both $\mathrm{Rh}$ and Ir were observed at three random locations (labelled as 


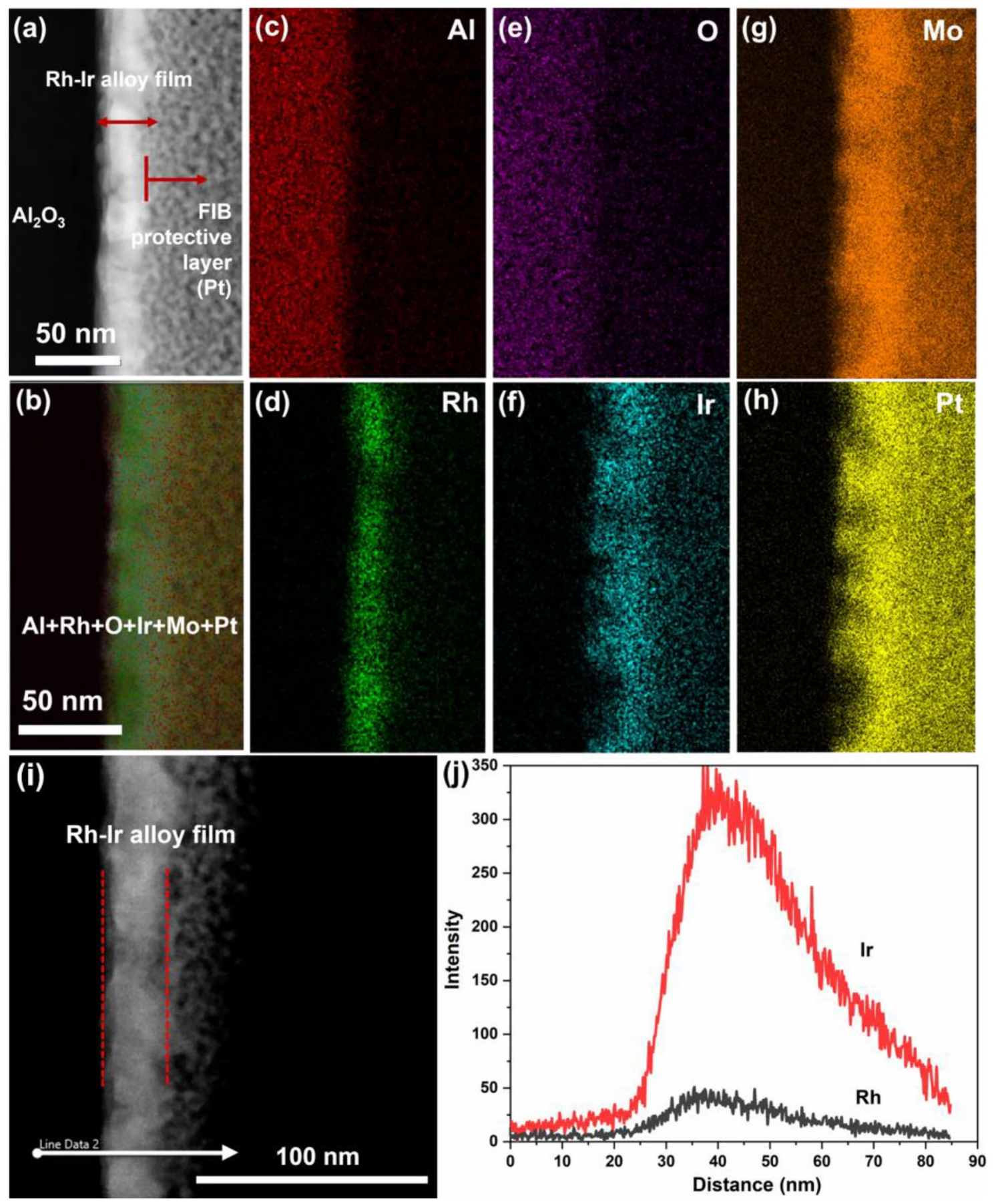

Figure 5. Cross-sectional TEM-EDX elemental mappings of the Rh-Ir alloy thin film. (a) Dark field STEM, (b) overlapping image, (c) Al ka1, (d) Rh La1, (e) O Ka1, (f) Ir Ma1, (g) Mo La1, (h) Pt Ma 1. (i) TEM image with a line indicating the EDX line scan position. (j) EDX line scan with Rh Ka and Ir Ka signals over a distance of $85 \mathrm{~nm}$ of the alloy thin film.

point 1, 2 and 3) in the metal film with consistent atomic ratio of $\sim 1.7$ (Fig. 4b), while no Rh or Ir was observed in the $\mathrm{Al}_{2} \mathrm{O}_{3}$ substrate region (points 4 and 5), confirming the diffusion of $\mathrm{Rh}$ and Ir.

The TEM cross-section in Fig. 5a shows a uniformly and conformal $\mathrm{Rh}$ - $\mathrm{Ir}$ alloy thin film on the $\mathrm{Al}_{2} \mathrm{O}_{3}$ substrate. The total thickness of the Rh-Ir alloy film was measured to be $30 \mathrm{~nm}$, which is in good agreement with that measured by XRR. EDS in mapping mode was used to examine the alloy thin film, indicating that Rh and Ir were relatively well-distributed evenly without obvious evidence of segregation (Fig. 5b-h).

From the STEM micrograph (Fig. 5a), we can observe some brightness variations across the film, which correspond to varying atomic weights. This shows that the alloying parameters have not been fully optimised to obtain full interdiffusion. As such, the EJH processing parameters would have to be studied and optimised to get complete interdiffusion. Nevertheless, it has been shown that alloying did take place to a significant degree. 

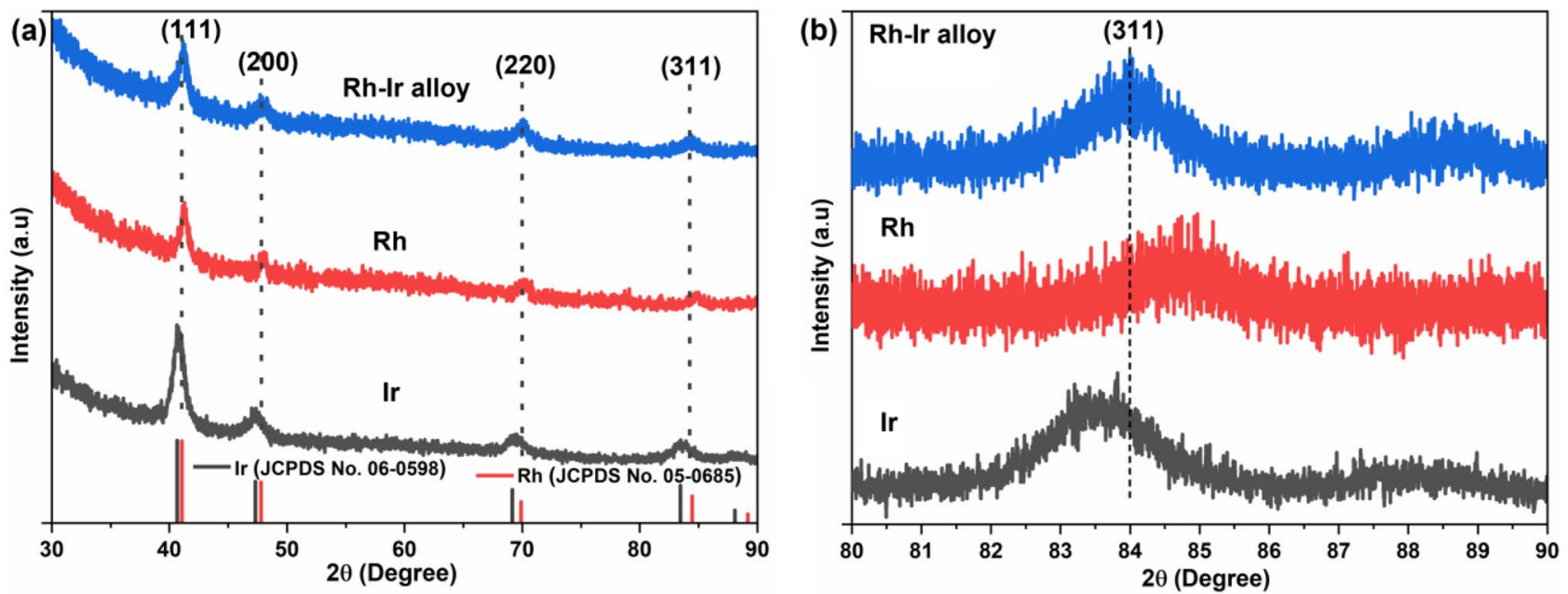

Figure 6. (a) XRD patterns of pure Ir film, pure Rh film and Rh-Ir thin film after EJH. (b) Slow scan XRD patterns of the (311) peak for pure Ir film, pure Rh film and Rh-Ir thin film after EJH.

It is noted to maximize the detection, the $\mathrm{X}$-ray detection range and energy resolution was limited to $10 \mathrm{keV}$ and divided to 1024 channels $(0.97 \mathrm{eV} /$ channel $)$. As a result, we are collecting Ma lines for Ir instead L-lines (Fig. 5f). Even with these settings, we still experienced Pt Ma interference ( $\Delta \mathrm{E}=97 \mathrm{eV}$ ) (Fig. 5h). Collecting Ir $\mathrm{La}$ is not preferred as it has much lower intensity, practically far from $\mathrm{Rh}$ La that necessitates to use larger energy range. Consequently, we reduced the energy resolutions.

Furthermore, the formation of Rh-Ir alloy film was also confirmed by TEM line analysis (Fig. 5i,j). The line scan was performed across the thin film and show the overlapping of Rh and Ir along a randomly chosen direction which can be assignable to the formation of alloy structure in the sintered sample ${ }^{36}$. The line intensity is not correlate directly to the element's concentration, as Ir and Rh has different X-ray emissions yield. We have not calibrated the X-ray signal to for full quantification.

Figure 6 shows X-ray diffraction patterns of synthesized pure Rh, Ir and Rh-Ir alloy. Four diffraction peaks are observed, corresponding to the (111), (200), (220) and (311) planes of Rh (JCPDS Card No.: 05-0685) and Ir (JCPDS Card No.: 06-0598) in all diffraction patterns ${ }^{37}$. This indicates that Rh-Ir alloy thin films adopted a face-centered cubic (FCC) structure which is consistent with both pure Rh and Ir thin film.

The peak broadening suggests that the crystallites are very small and finely dispersed. The peak corresponding to the (311) plane for pure $\mathrm{Rh}$ is observed at $2 \theta=84.5^{\circ}$ and that for pure Ir is observed at $2 \theta=83.5^{\circ}$. The peak corresponding to (311) plane for Rh-Ir after EJH is observed at $2 \theta=84.0^{\circ}$ as shown in Fig. 6 . The shift in (311) diffraction peak in $\mathrm{Rh}$-Ir alloy thin film confirms the formation of $\mathrm{Rh}$-Ir alloy after $\mathrm{EJH}^{38}$. All these peak shifts were also reported by Sampath et al. in the formation of Ir-Rh bimetallic alloy nanoparticles made by hydrothermal method ${ }^{38}$.

High resolution XPS spectra Rh $3 \mathrm{~d}$ and $\mathrm{Ir} 4 \mathrm{f}$ were collected to investigate the local bonding structure and chemical states of the alloy thin film as shown in Fig. 7. The binding energy values of $308.0 \mathrm{eV}$ and $312.8 \mathrm{eV}$ represent $\mathrm{Rh} 3 \mathrm{~d}_{5 / 2}$ and $\mathrm{Rh} 3 \mathrm{~d}_{3 / 2}$ levels of pure $\mathrm{Rh}$ in metallic state which is similar with the bonding energy, observed in alloy sample, namely $307.8 \mathrm{eV}$ and $312.6 \mathrm{eV}$. The spin separations of the $\mathrm{Rh} 3 \mathrm{~d}$ for both pure $\mathrm{Rh}$ and alloyed $\mathrm{Rh}-\mathrm{Ir}$ are measured to be $3.0 \mathrm{eV}$ which is characteristic of the metallic Rh. The intensity at $312 \mathrm{eV}$ comes from the overlap of $\mathrm{Rh} 3 \mathrm{~d}_{3 / 2}$ with $\operatorname{Ir} 4 \mathrm{~d}_{3 / 2}^{39,40}$. The other values observed at 309 and $314 \mathrm{eV}$ for pure and alloyed samples are consistent with the oxidized species which are attributed to surface oxidation under ambient conditions. The relative ratios reveal that $\mathrm{Rh}$ is majority composition in both pure and alloy samples (Fig. 7a,b). Ir spectra for pure Ir and Rh-Ir alloy are shown in Fig. 7c,d. the spectra indicate the presence of metallic Ir with a binding energy of 64.5 and $61.5 \mathrm{eV}$ for pure Ir and at $64.4 \mathrm{eV}$ and $61.4 \mathrm{eV}$ for Rh-Ir alloy. Ir $5 \mathrm{p}_{1 / 2}$ peak is also observed at $63.1 \mathrm{eV}$ which overlaps with the Ir $4 \mathrm{f} \mathrm{peaks}^{40}$. The relative ratios reveal that metallic Ir is dominant compared to oxides for both Ir and alloy. No significant variation in the intensity of the oxide component was found in Ir before and after alloying. It is worth noting that $\mathrm{Rh}$ as the first layer on $\mathrm{Al}_{2} \mathrm{O}_{3}$ was not detected in $\mathrm{Rh}$-Ir thin film before EJH, which was only observed in the sample after EJH, implying the formation of alloy after EJH.

From the SEM image (Fig. 2a), it can be seen that the Rh-Ir thin film is constituted by nanoparticles, where $\mathrm{Rh}$ is the base layer and Ir is the surface layer. Based on this, MD simulation was carried out using Rh-core @ Ir-shell nanoparticle (Rh-Ir NP) model to further explore the alloying mechanism. Note that melting points of bulk iridium and rhodium of the EAM potential are $2007 \pm 15$ and $1787 \pm 15^{\circ} \mathrm{C}$, which are lower than the experimental values $\left(2466\right.$, and $1964^{\circ} \mathrm{C}$, respectively) ${ }^{41}$. Therefore, the melting point of the Rh-Ir NP will be estimated lower than that of the experimental value in scale. Snapshots of Rh-Ir NP taken at four representative temperatures were extracted from $\mathrm{MD}$ simulations of the heating process, as shown in Fig. 8. The Lindemann index is illustrated on its right side. At $27^{\circ} \mathrm{C}$, the atoms in the Rh-Ir NP are orderly arranged. When the temperature increases to $1127^{\circ} \mathrm{C}$, only surface pre-melting of Ir shell occurs. Also, Rh core is highly ordered. When the temperature is $1327^{\circ} \mathrm{C}$, Ir melts and diffuses into the Rh core since the thickness of Ir shell is thin. Meanwhile, the internal $\mathrm{Rh}$ atoms are still in order. As the temperature reaches $1347^{\circ} \mathrm{C}$, an instantaneous accomplishment 

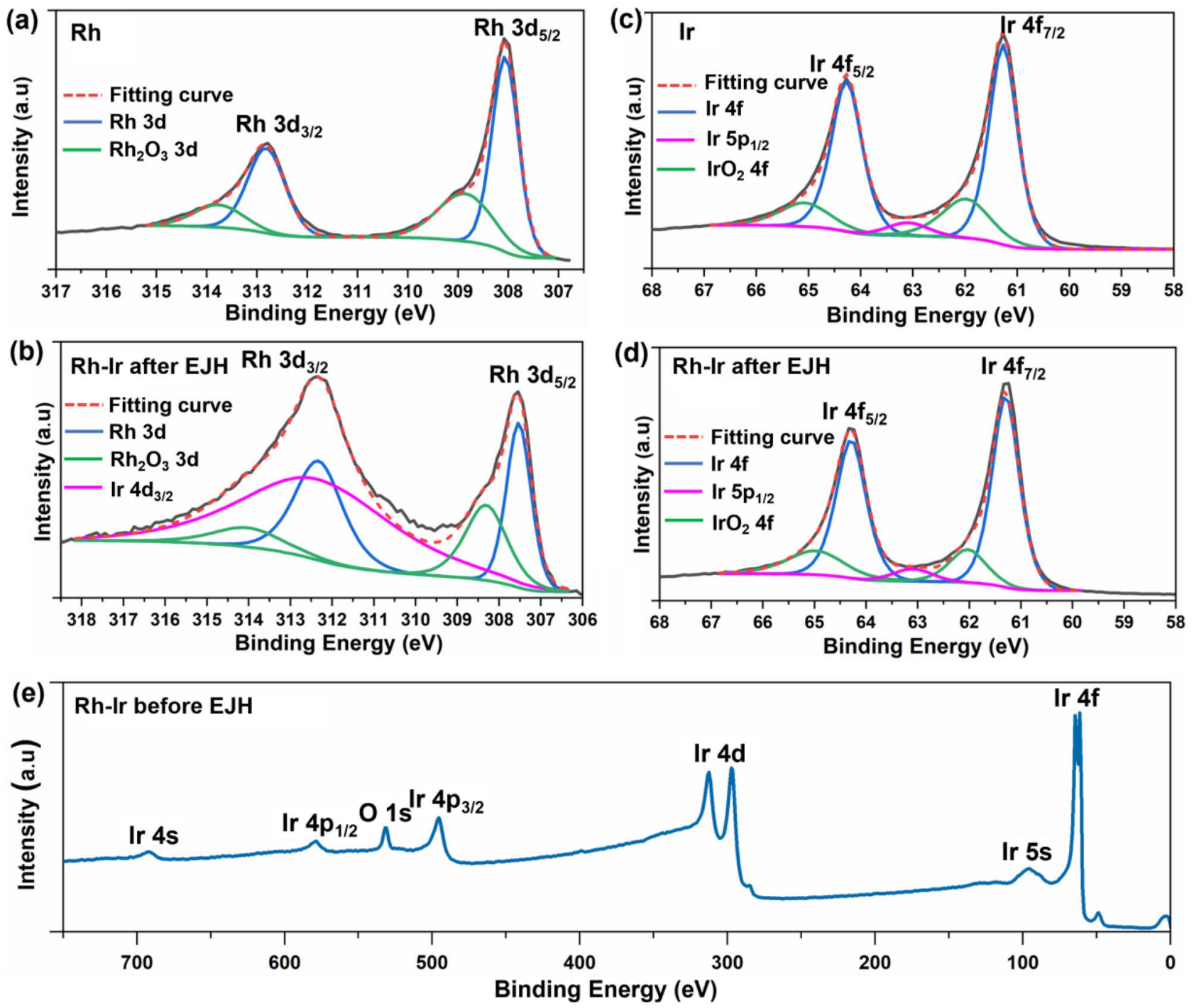

Figure 7. Rh $3 \mathrm{~d}$ XPS spectra of (a) pure Rh and (b) Rh-Ir after EJH. Ir 4f XPS spectra of (c) pure Ir and (d) Rh-Ir after EJH. (e) XPS survey spectra of Rh-Ir thin film before EJH.

(a)

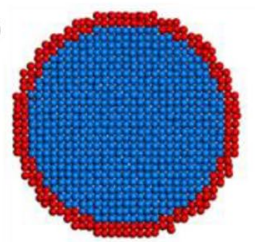

$27^{\circ} \mathrm{C}$

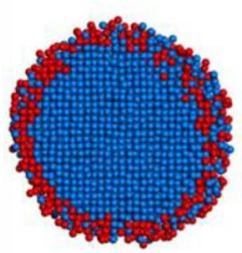

$1327^{\circ} \mathrm{C}$

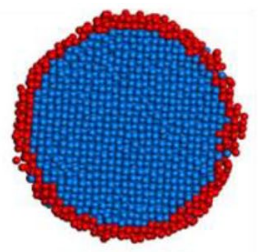

$1127^{\circ} \mathrm{C}$

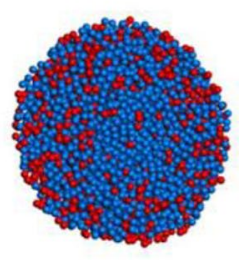

$1347^{\circ} \mathrm{C}$ (b)

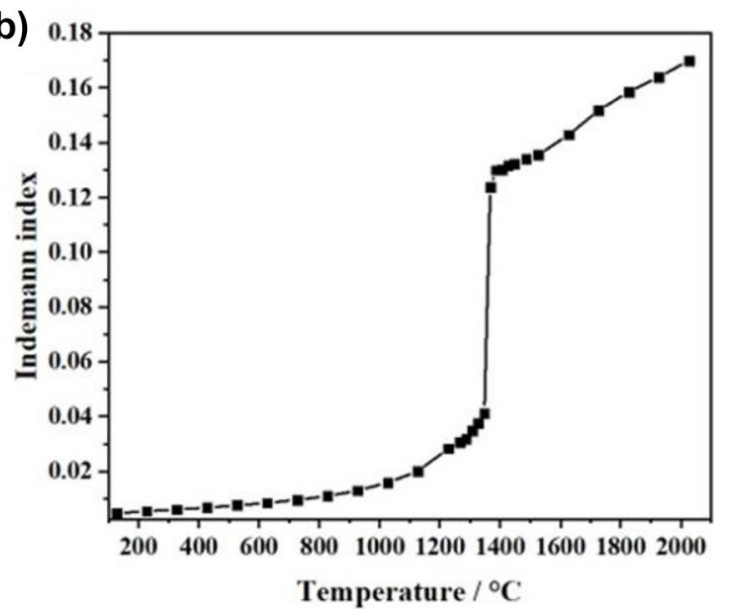

Figure 8. (a) Snapshots of Rh-Ir NP taken at four representative temperatures during the heating process and (b) the Lindemann indices. Coloring denotes type of atom: red, Ir atom; blue, Rh atom. 
of the solid-liquid phase transition of the Rh core occurs. As a result, the alloying of the Rh-Ir NP experiences a quite narrow temperature interval $\left(20^{\circ} \mathrm{C}\right.$, from 1327 to $\left.1347^{\circ} \mathrm{C}\right)$. Subsequently, Ir atoms continue diffusion into the $\mathrm{Rh}$ layer and both metal atoms are distributed uniformly, which was in agreement with elemental mapping using TEM (Fig. 5). However, the simulated temperature at which $\mathrm{Rh}-\mathrm{Ir}$ alloy formed $\left(1347^{\circ} \mathrm{C}\right)$ is $\sim 267^{\circ} \mathrm{C}$ higher than the experimental one $\left(1080^{\circ} \mathrm{C}\right)$. The possible reasons include: (1) It has been reported that the impurities could lower the melting point of crystals. Noble-metal ALD processes usually produce very pure films with low impurity contents ${ }^{42}$. However, during our experiment, small amount of Rh oxide was observed by XPS. So it is possible the melting point of the Rh-Ir thin film was reduced because of the impurities (metal oxides) in the deposited films ${ }^{43,44}$. (2) As reported in our previous work, some ligand remained in the as deposited film from $\mathrm{Rh}$ precursor $\left(\mathrm{Rh}(\mathrm{acac})_{3}\right)$, ALD Rh precursors are likely amorphous with lower melting temperatures than the crystalline counterparts ${ }^{23,45,46}$.

\section{Conclusion}

In conclusion, ALD technique is capable of fabricate relatively uniform and continuous sub-50-nm thick alloy thin film on 2D substrates compared to other alloy synthesis methods. Our initial work focused on Rh-Ir alloy thin film synthesis involving ALD and EJH alloying techniques. SEM and TEM reveal both Rh and Ir were distributed uniformly in the single-phase solid-solution alloy film with no signs of macroscopic phase segregation on $\mathrm{Al}_{2} \mathrm{O}_{3}$ substrate similar to bulk Rh-Ir alloys prepared by other methods. Optimization of the CTS parameters will be the subject of ongoing works. MD simulations was also applied to investigate the melting mechanism of $\mathrm{Rh}$-Ir thin film. The pre-melting temperature of Ir on the Rh-Ir surface was determined before alloying. As the temperature increase, Ir atoms diffuse into $\mathrm{Rh}$ layer, forming a $\mathrm{Rh}-\mathrm{Ir}$ alloy which is basically in agreement with the experimental characterization results of Rh-Ir alloy thin film. In summary, our proof-of-concept experiments suggests coupling ALD with EJH enables a viable approach to prepare homogenous single-phase solid-solution metal alloy thin films that may find use for various applications in catalysis, microelectronics and other fields.

Received: 1 October 2021; Accepted: 25 January 2022

Published online: 15 February 2022

\section{References}

1. Wei, Y. L. et al. A measurement system of high-temperature oxidation environment with ultrasonic $\mathrm{Ir}_{0.6} \mathrm{Rth}_{0.4}$ alloy thermometry. Ultrasonics 89, 102-109 (2018).

2. Shi, Y. Z., Yang, B. \& Liaw, P. K. Corrosion-resistant high-entropy alloys A review. Metals 7, 43 (2017).

3. Wang, Z. et al. High hardness and fatigue resistance of CoCrFeMnNi high entropy alloy films with ultrahigh-density nanotwins. Int. J. Plast. 131, $102726(2020)$.

4. Yamabe-Mitarai, Y., Ro, Y., Harada, H. \& Maruko, T. Ir-base refractory superalloys for ultra-high temperatures. Metall. Mater. Trans. A 29, 537-549 (1998).

5. Choi, J. H., Park, K. W., Park, I. S., Nam, W. H. \& Sung, Y. E. Methanol electro-oxidation and direct methanol fuel cell using Pt/ $\mathrm{Rh}$ and $\mathrm{Pt} / \mathrm{Ru} / \mathrm{Rh}$ alloy catalysts. Electrochim. Acta 50, 787-790 (2004).

6. Wang, H. S. \& Abruña, H. D. Rh and Rh alloy nanoparticles as highly active $\mathrm{H}_{2}$ oxidation catalysts for alkaline fuel cells. ACS Catal. 9, 5057-5062 (2019).

7. Niu, J. L. et al. Research on a $\mathrm{Zn}-\mathrm{Cu}$ alloy as a biodegradable material for potential vascular stents application. Mater. Sci. Eng. C 69, 407-413 (2016).

8. Li, P. J., Zhang, Y. G., Yang, X. G., Gao, Y. H. \& Ge, S. X. Alloyed PtNi counter electrodes for high-performance dye-sensitized solar cell applications. J. Alloys Compd. 725, 1272-1281 (2017).

9. Hadef, $\mathrm{F}$. Synthesis and disordering of $\mathrm{B} 2 \mathrm{TM}-\mathrm{Al}(\mathrm{TM}=\mathrm{Fe}, \mathrm{Ni}, \mathrm{Co})$ intermetallic alloys by high energy ball milling: A review. Powder Technol. 311, 556-578 (2017).

10. Sharma, N., Jangra, K. K. \& Raj, T. Fabrication of NiTi alloy: A review. Proc. Inst. Mech. Eng. L 232, 250-269 (2018).

11. Miikkulainen, V., Leskelä, M., Ritala, M. \& Puurunen, R. L. Crystallinity of inorganic films grown by atomic layer deposition: Overview and general trends. J. Appl. Phys. 113, 2 (2013).

12. Knez, M., Nielsch, K. \& Niinistö, L. Synthesis and surface engineering of complex nanostructures by atomic layer deposition. Adv. Mater. 19, 3425-3438 (2007)

13. Christensen, S. T. \& Elam, J. W. Atomic layer deposition of Ir-Pt alloy films. Chem. Mater. 22, 2517-2525 (2010).

14. Lu, J. et al. Toward atomically-precise synthesis of supported bimetallic nanoparticles using atomic layer deposition. Nat. Commun. 5, 3264 (2014).

15. Shimizu, H., Shima, K., Suzuki, Y., Momose, T. \& Shimogaki, Y. Precursor-based designs of nano-structures and their processing for $\mathrm{Co}(\mathrm{W})$ alloy films as a single layered barrier/liner layer in future $\mathrm{Cu}$-interconnect. J. Mater. Chem. C 3, 2500-2510 (2015).

16. Lee, H.-J., Hong, T. E. \& Kim, S.-H. Atomic layer deposited self-forming Ru-Mn diffusion barrier for seedless Cu interconnects. J. Alloys Compd. 686, 1025-1031 (2016).

17. Ahn, J., Ahn, C., Jeon, S. \& Park, J. Atomic layer deposition of inorganic thin films on 3D polymer nanonetworks. Appl. Sci. 9 , 1990 (2019).

18. Schwille, M. C. et al. Experimental and simulation approach for process optimization of atomic layer deposited thin films in high aspect ratio 3D structures. J. Vac. Sci. Technol. A 35, 01B118 (2017).

19. Wang, C. G. et al. A general method to synthesize and sinter bulk ceramics in seconds. Science 368, 521-526 (2020).

20. Yao, Y. et al. Carbothermal shock synthesis of high-entropy-alloy nanoparticles. Science 359, 1489-1494 (2018).

21. Shibata, T. et al. Size-dependent spontaneous alloying of Au-Ag nanoparticles. J. Am. Chem. Soc. 124, 11989-11996 (2002).

22. Guisbiers, G., Abudukelimu, G. \& Hourlier, D. Size-dependent catalytic and melting properties of platinum-palladium nanoparticles. Nanoscale Res. Lett. 6, 1-5 (2011).

23. Zou, Y. M. et al. Atomic layer deposition of palladium and rhodium thin film using low-concentration ozone. RSC Adv. 11, 22773-22779 (2021).

24. Knapas, K. \& Ritala, M. In situ reaction mechanism studies on atomic layer deposition of $\mathrm{Ir}$ and $\mathrm{IrO}_{2}$ from $\mathrm{Ir}(\mathrm{acac})_{3}$. Chem. Mater. 23, 2766-2771 (2021).

25. Luong, D. X. et al. Gram-scale bottom-up flash graphene synthesis. Nature 577, 647-651 (2020).

26. Plimpton, S. Fast parallel algorithms for short-range molecular dynamics. J. Comput. Phys. 117, 1-19 (1995).

27. Plimpton, S. J. \& Thompson, A. P. Computational aspects of many-body potentials. MRS Bull. 37, 513-521 (2012). 
28. Sheng, H. W., Kramer, M. J., Cadien, A., Fujita, T. \& Chen, M. W. Highly optimized embedded-atom-method potentials for fourteen FCC metals. Phys. Rev. B 83, 134118 (2011).

29. Daw, M. S. \& Baskes, M. I. Embedded-atom method: Derivation and application to impurities, surfaces, and other defects in metals. Phys. Rev. B 29, 6443 (1984).

30. Evans, D. J. \& Holian, B. L. The Nose-Hoover thermostat. J. Chem. Phys. 83, 4069-4074 (1985).

31. Zhou, Y. Q., Karplus, M., Ball, K. D. \& Berry, R. S. The distance fluctuation criterion for melting: Comparison of square-well and Morse potential models for clusters and homopolymers. J. Chem. Phys. 116, 2323-2329 (2002).

32. Yao, X. Z., Wei, Y. P., Wang, Z. X. \& Gan, L. Revealing the role of surface composition on the particle mobility and coalescence of carbon-supported Pt alloy fuel cell catalysts by in situ heating (S) TEM. ACS Catal. 10, 7381-7388 (2020).

33. Yang, Y. et al. Rapid laser pulse synthesis of supported metal nanoclusters with kinetically tunable size and surface density for electrocatalytic hydrogen evolution. ACS Appl. Nano Mater. 3, 2959-2968 (2020).

34. Drouin, D. et al. CASINO V2. 42-A fast and easy-to-use modeling tool for scanning electron microscopy and microanalysis users. Scanning 29, 92-101 (2007).

35. Bharadwaj, S. R., Tripathi, S. N. \& Chandrasekharaiah, M. S. The Ir-Pt (iridium-platinum) system. J. Phase Equilib. 16, 460-464 (1995).

36. Guo, H. Y. et al. Rational design of rhodium-iridium alloy nanoparticles as highly active catalysts for acidic oxygen evolution. ACS Nano 13, 13225-13234 (2019).

37. Le Drogoff, B., El Khakani, M. A., Silva, P. R. M., Chaker, M. \& Ross, G. G. Surface properties of pulsed laser deposited Ir, Rh, and $\mathrm{Ir}_{0.9} \mathrm{Rh}_{0.1}$ thin films for use as microelectrode arrays in electroanalytical heavy metal trace sensors. Appl. Surf. Sci. 152, 77-84 (1999).

38. Kiran, V. et al. Electro-oxidation of borohydride on rhodium, iridium, and rhodium-iridium bimetallic nanoparticles with implications to direct borohydride fuel cells. J. Electrochem. Soc. 157, B1201 (2010).

39. Silva, J. C. M. et al. Iridium-rhodium nanoparticles for ammonia oxidation: Electrochemical and fuel cell studies. ChemElectroChem 4, 1101-1107 (2017)

40. Freakley, S. J., Ruiz-Esquius, J. \& Morgan, D. J. The X-ray photoelectron spectra of $\mathrm{Ir}, \mathrm{I} \mathrm{rO}_{2}$ and $\mathrm{IrCl}_{3}$ revisited. Surf. Interface Anal. 49, 794-799 (2017).

41. Brandes, E. A. \& Brook, G. B. Smithells Metals Reference Book (Butterworth, 1983).

42. Hämäläinen, J., Ritala, M. \& Leskelä, M. Atomic layer deposition of noble metals and their oxides. Chem. Mater. 26, 786-801 (2014).

43. Mottet, C., Rossi, G., Baletto, F. \& Ferrando, R. Single impurity effect on the melting of nanoclusters. Phys. Rev. Lett. 95, 035501 (2005).

44. Lyalin, A., Hussien, A., Solov'yov, A. V. \& Greiner, W. Impurity effect on the melting of nickel clusters as seen via molecular dynamics simulations. Phys. Rev. B 79, 165403 (2009).

45. Thompson, M. O. et al. Melting temperature and explosive crystallization of amorphous silicon during pulsed laser irradiation. Phys. Rev. Lett. 52, 2360 (1984).

46. Tan, K. W. et al. Transient laser heating induced hierarchical porous structures from block copolymer-directed self-assembly. Science 349, 54-58 (2015).

\section{Acknowledgements}

The work was supported by the Agency for Science, Technology and Research (A*STAR) under award number SERC A1983c0032, AME Individual Research Grant (IRG) for this project.

\section{Author contributions}

A.T. and S.L. conceived the project. Y.G. wrote this paper. A.T., S.L., K.T. revised the manuscript. Y.G. and Y.Z. designed, finished the ALD experiment and characterizations. C.C. accomplished simulation work. L.W. and K. T. conducted the EJH experiment. R.M., R.G. contributed to data collection. All authors discussed the results and contributed to the manuscript.

\section{Competing interests}

The authors declare no competing interests.

\section{Additional information}

Correspondence and requests for materials should be addressed to S.L. or A.I.Y.T.

Reprints and permissions information is available at www.nature.com/reprints.

Publisher's note Springer Nature remains neutral with regard to jurisdictional claims in published maps and institutional affiliations.

(c) (i) Open Access This article is licensed under a Creative Commons Attribution 4.0 International License, which permits use, sharing, adaptation, distribution and reproduction in any medium or format, as long as you give appropriate credit to the original author(s) and the source, provide a link to the Creative Commons licence, and indicate if changes were made. The images or other third party material in this article are included in the article's Creative Commons licence, unless indicated otherwise in a credit line to the material. If material is not included in the article's Creative Commons licence and your intended use is not permitted by statutory regulation or exceeds the permitted use, you will need to obtain permission directly from the copyright holder. To view a copy of this licence, visit http://creativecommons.org/licenses/by/4.0/.

(C) The Author(s) 2022 